\title{
Report of a rare case of histiocytic necrotizing lymphadenitis with bilateral pleural effusion diagnosed via cervical lymph node biopsy
}

\author{
Xuchun Liu', Shubin Huang", Guohua Jiang"II \\ Chizhou People's Hospital, Chizhou, Anhui, China
}

'MD. Specialist, Department of Respiratory Medicine, Chizhou People's Hospital, Chizhou, Anhui, China.

"MD. Specialist, Department of Pathology, Chizhou People's Hospital, Chizhou, Anhui, China.

'"MD, PhD. Professor, Department of Respiratory Medicine, Chizhou People's Hospital, Chizhou, Anhui, China.

\section{KEY WORDS:}

Histiocytic necrotizing lymphadenitis.

Pleural effusion.

Tuberculosis.

Lymph nodes

Biopsy.

\section{PALAVRAS-CHAVE:}

Linfadenite histiocítica necrosante.

Derrame pleural.

Tuberculose.

Linfonodos.

Biópsia.

\begin{abstract}
CONTEXT: Histiocytic necrotizing lymphadenitis $(\mathrm{HNL})$ is a rare disorder that is often benign and selflimiting. There have been reports of co-occurrence of HNL with other diseases, including systemic lupus erythematosus, hemophagocytic syndrome and antiphospholipid syndrome.

CASE REPORT: Here, we report a case in which a patient experienced unexplained fever, swelling of the cervical lymph node and bilateral pleural effusion and was ultimately diagnosed with HNL based on results from a lymph node biopsy. After treatment with glucocorticoid, the patient regained normal body temperature, the swelling of the lymph nodes disappeared and the pleural effusion was reabsorbed. CONCLUSIONS: The pathogenesis of HNL remains unclear, and pleural effusion is rarely reported in HNL patients. We presented this case to improve diagnostic awareness of this condition among clinicians and help reduce the likelihood of misdiagnosis.
\end{abstract}

\section{INTRODUCTION}

Histiocytic necrotizing lymphadenitis (HNL) is a rare disorder, and its pathogenesis remains unclear. Some scholars believe that HNL is not an independent disease and that it may be a manifestation of an underlying autoimmune disorder; as such, the diagnosis and treatment for HNL should be targeted to the underlying disease instead. There have been reports of co-occurrence of HNL with other diseases, including systemic lupus erythematosus (SLE), hemophagocytic syndrome and antiphospholipid syndrome. ${ }^{1,2}$

However, pleural effusion has rarely been reported in HNL patients. Here, we report a case in which the patient experienced unexplained fever, swelling of the cervical lymph node and bilateral pleural effusion and was ultimately diagnosed with HNL based on the results from a lymph node biopsy.

\section{CASE REPORT}

A 26-year-old woman was hospitalized due to anorexia and fatigue that had lasted one month. Ten days before admission, the patient began to experience intermittent chills and a fever between 38.5 and $40{ }^{\circ} \mathrm{C}$. Physical examination on admission revealed that both cervical lymph nodes were swollen and tender, more obviously so on the left side. Double lung auscultation detected lower breathing sounds.

Laboratory examinations revealed the following: white blood cell count, $2.45 \times 10^{9} / 1$; neutrophils: 48.70\%; lymphocytes: $42 \%$; red blood cell count: $3.9 \times 10^{12} / 1$; hemoglobin: $101 \mathrm{~g} / \mathrm{l}$; platelets: $141 \times 10^{9} / \mathrm{l}$; erythrocyte sedimentation rate: $30 \mathrm{~mm} / \mathrm{h}$; procalcitonin: $0.095 \mathrm{ng} / \mathrm{ml}$; and C-reactive protein: $19.11 \mathrm{mg} / \mathrm{l}$. The results for tumor markers, autoimmune antibodies, anti-neutrophil cytoplasmic antibodies and blood cultures were normal. The T-SPOT and purified protein derivative tests for tuberculosis were negative, as were tests for pleural tuberculosis antibodies. Bone marrow cytological analysis revealed bone marrow hyperplasia.

Computed tomography (CT) of the chest revealed slight swelling of the mediastinal lymph nodes and a small amount of bilateral pleural effusion, which was slightly more severe on the 
right side (Figure 1). Ultrasonography of the neck revealed multiple swollen bilateral supraclavicular lymph nodes, with some as large as $16 \times 7 \mathrm{~mm}$. Ultrasonography of the upper abdomen and uterus did not reveal any anomaly.

The pleural puncture technique was applied to drain excessive fluid, and Rivalta test results were positive. The pleural effusion $\mathrm{pH}$ was 7.35 (normal, 6.8-7.6), proteins were $32 \mathrm{~g} / \mathrm{l}$ (normal, 0-30), and lactate dehydrogenase, adenosine deaminase and carcinoembryonic antigen levels were $2186 \mathrm{U} / \mathrm{l}$ (normal, 0-200), $30 \mathrm{U} / \mathrm{l}$ (normal, 0-40) and $1.64 \mathrm{ng} / \mathrm{ml}$ (normal, 0-6.5), respectively. The total number of nucleated cells in the pleural effusion was $3886 \times 10^{6} / 1$ (normal, 100$\left.500 \times 10^{6} / 1\right)$. Repeated pleural fluid cytological analysis revealed a large quantity of lymphocytes. Pleural fluid cultures were negative.

The patient underwent two weeks of anti-inflammatory treatment with $0.2 \%$ levofloxacin, $300 \mathrm{ml}$ daily administered by means of intravenous drip, which was ineffective. She still experienced intermittent fever, and ultrasonography revealed continued pleural effusion.

A biopsy on the left cervical lymph node showed scattered fibrin deposition, a large amount of nuclear debris and large mononuclear cell aggregates in the necrotic area (Figure 2). The pathological diagnosis was histiocytic necrotizing lymphadenitis.

Antibiotics were discontinued after two weeks of treatment with intravenous infusion of levofloxacin, and $10 \mathrm{mg}$ of oral prednisone was administered three times daily. Within three days, the patient's body temperature gradually returned to normal. After general improvement during a one-week observation period, she was discharged and was prescribed a reduced dose of oral prednisone. Three months later, a chest radiograph showed that the double lung texture disorder and bilateral pleural effusion had disappeared (Figure 3).

\section{DISCUSSION}

Histiocytic necrotizing lymphadenitis is a rare inflammatory disease of the lymph nodes. It is more common in young women and has a natural course of 1-4 months, ${ }^{3}$ but relapse may occur. The cause of HNL remains unclear, but it is probably associated with infections and autoimmune disorders. A variety of viruses, such as adenovirus, Epstein-Barr virus and herpes virus, are reportedly associated with the disease. HNL onset is commonly associated with acute or subacute clinical manifestations, such as swollen lymph nodes, fever and neutropenia. The most commonly affected lymph nodes are cervical, followed by axillary. Intermittent fever, fatigue, joint pain, hepatosplenomegaly and skin rashes are also common symptoms. Rarely, HNL patients exhibit complex clinical manifestations involving multiple organ systems, similar to those for rheumatic diseases.

It has been reported in the literature that the clinical manifestations of HNL are similar to those of certain connective tissue diseases, such as adult-onset Still's disease and SLE. There are also reports of HNL co-occurring with these diseases, with the strongest link to SLE. Reports have suggested that HNL can occur before, after or concurrently with SLE pathogenesis. ${ }^{4}$ In particular,

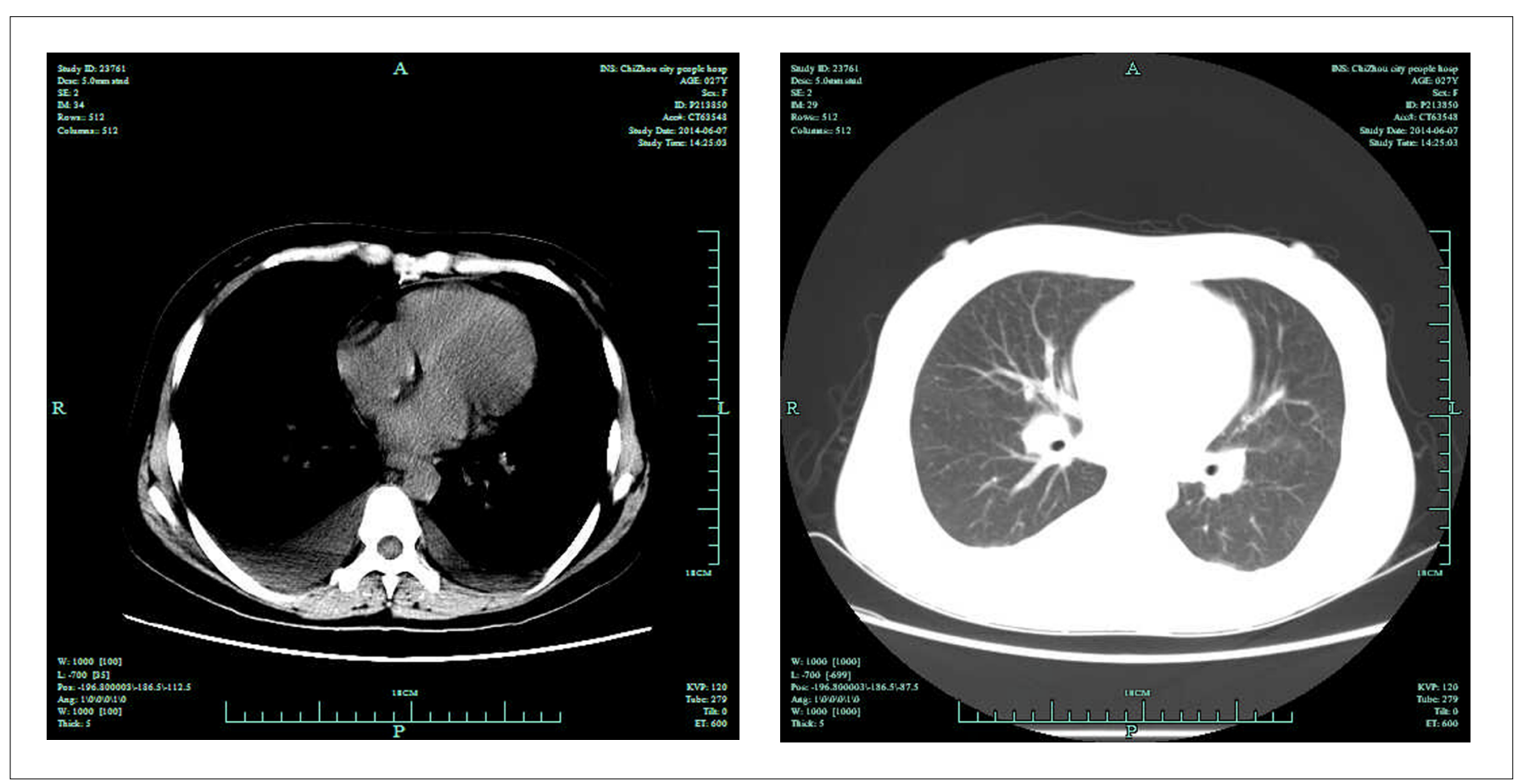

Figure 1. Computed tomography of the chest, revealing slight swelling of the mediastinal lymph nodes and a small amount of bilateral pleural effusion, with slightly more on the right side than on the left. 
anti-nuclear antibody-positive HNL is more likely to develop into SLE, and certain scholars even hold that HNL is a unique manifestation of SLE. ${ }^{5}$ Additionally, certain clinical manifestations of lymphoma are very similar to those of HNL; as such, HNL patients are often misdiagnosed as having malignant lymphoma.

Noninvasive diagnostic methods are limited in their effectiveness. At present, application of 18F-deoxyglucose (18F-FDG) positron emission tomography (PET)/CT is widely used as a tumor metabolism imaging technique for determining lymph node malignancy. However, studies using FDG imaging and PET/CT have suggested that in HNL patients, lymph node uptake values ranged from 2.05 to 13.94 , with an average of $6.25 \pm 3.32$. Cervical lymph nodes often have a higher uptake value. Evidently, PET/CT scans alone are not sufficient to differentiate between HNL and lymphoma, but the biopsy site can be determined using the standard uptake value. ${ }^{6}$

Pathological and immunohistochemical examinations are important tools for differential diagnosis. In 1995, $\mathrm{Kuo}^{7}$ further defined HNL into three subtypes: proliferative, necrotic and xanthoma-like, which may reflect the different stages of disease development, namely hyperplasia-necrosis-xanthoma or granulationreabsorption-recovery. The pathological characteristics of HNL include a patchy lymph node disorder, commonly found in the juxtacortical area with visible fibrinoid necrosis and a large amount of nuclear debris. Necrotic areas are surrounded by proliferative cells, but granulocytes and plasma cells are rare or absent. Specialized or tufted CD68-positive and MPO-positive cells are important immunohistochemical features of $\mathrm{HNL} .{ }^{8} \mathrm{~T}$-cells are the most commonly found lymphocytes, while B-cells and natural killer cells are rarer. Using an electron microscope, a large number of

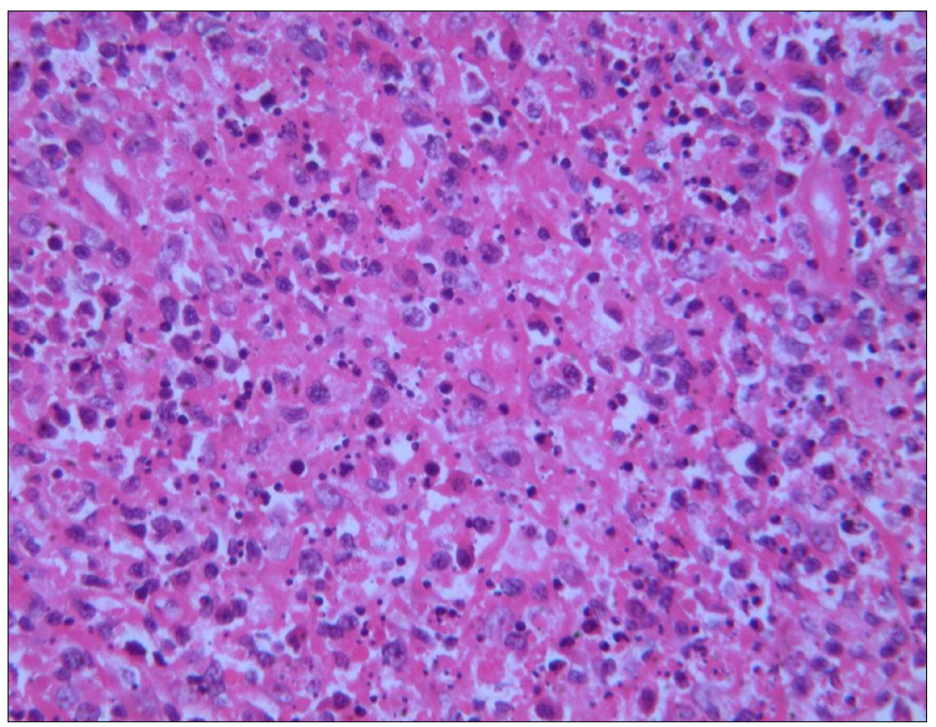

Figure 2. Biopsy specimen from the left cervical lymph node. Pathological manifestation showing fibrin deposition, a large amount of nuclear debris and large mononuclear cell aggregates within the necrotic regions (hematoxylin and eosin staining; magnification $\times 400$ ). apoptotic bodies surrounded by mononuclear histiocytes and scattered T-cells undergoing apoptosis were found in the lymph nodes of HNL patients. These three observations are closely related, and they suggest that T-lymphocyte apoptosis may play an important role in the pathogenesis of HNL. ${ }^{9}$

The incidence of pleural effusion in HNL is low and rarely reported clinically, so we cannot reach any conclusion regarding the likelihood that HNL patients might have pleural effusion. In the present case, the patient had an exudate. The mechanism of its occurrence remains unclear, and it is possible that the disease itself may lead to pleural disruption, thus causing exudative pleurisy; however, there is a lack of histological evidence to support this concept. Pleural biopsies can easily miss the location of the lesion, since they can only collect a relatively small amount of the sample, and they may be prone to false negative results. On the other hand, thoracoscopy or thoracotomy examinations are highly invasive and are not very popular with most patients. Additionally, the possibility that HNL might be caused by an endogenous sex hormone dysfunction cannot be ruled out, since corticosteroid therapy has been observed to lead to complete reabsorption of pleural effusion. The mechanism of pleural effusion in HNL patients still need to be further investigated.

We searched for similar cases in different databases (PubMed, Embase and LILACS) using the following terms: "histiocytic necrotizing lymphadenitis" AND "pleural effusion" or "Kikuchi-Fujimoto disease" AND "pleural effusion" (Table 1). We found that only one case had been published. ${ }^{10}$

The treatments for HNL include non-steroidal anti-inflammatory drugs and corticosteroids; antibiotic treatment is often

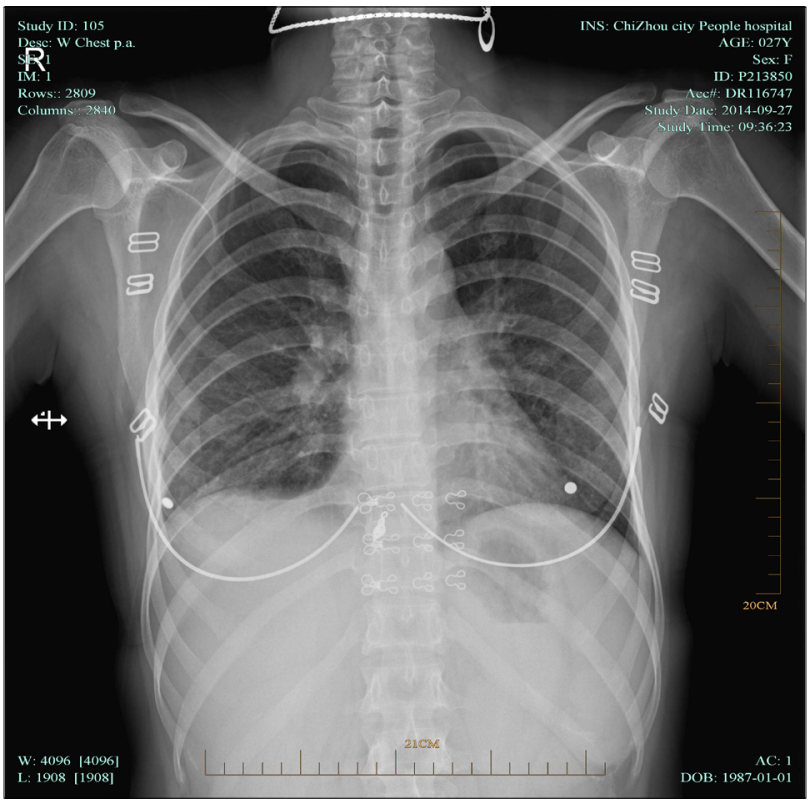

Figure 3. Chest radiograph, showing that the double lung texture disorder and bilateral pleural effusion had disappeared. 
Table 1. Results from a search of the literature in medical databases, for case reports on "histiocytic necrotizing lymphadenitis with bilateral pleural effusion diagnosed via cervical lymph node biopsy". The search was conducted on December 22, 2016

\begin{tabular}{|c|c|c|c|}
\hline Database & Search strategies & $\begin{array}{l}\text { Papers } \\
\text { found }\end{array}$ & $\begin{array}{l}\text { Related } \\
\text { papers }\end{array}$ \\
\hline $\begin{array}{l}\text { MEDLINE } \\
\text { (via PubMed) }\end{array}$ & $\begin{array}{l}\text { ((histiocytic necrotizing } \\
\text { lymphadenitis[Title]) AND } \\
\text { pleural effusion[Title]) }\end{array}$ & 0 & 0 \\
\hline $\begin{array}{l}\text { MEDLINE } \\
\text { (via PubMed) }\end{array}$ & $\begin{array}{c}\text { ((Kikuchi-Fujimoto } \\
\text { disease[Title]) } \\
\text { AND pleural effusion[Title]) }\end{array}$ & 1 & 1 \\
\hline $\begin{array}{l}\text { Embase } \\
\text { (via Elsevier) }\end{array}$ & $\begin{array}{l}\text { ((histiocytic necrotizing } \\
\text { lymphadenitis[Title]) AND } \\
\text { pleural effusion[Title]) }\end{array}$ & 0 & 0 \\
\hline $\begin{array}{l}\text { LILACS } \\
\text { (via BVS) }\end{array}$ & $\begin{array}{l}\text { ((histiocytic necrotizing } \\
\text { lymphadenitis[Title]) AND } \\
\text { pleural effusion[Title]) }\end{array}$ & 0 & 0 \\
\hline
\end{tabular}

ineffective. Although non-steroidal anti-inflammatory drugs can elicit partial effects, there have been reports suggesting that this treatment may cause patients to be more prone to relapse. ${ }^{11}$ It has been suggested that early glucocorticoid therapy should be used to treat HNL, and that the duration and intensity of the treatment should be based on the patient's response to hormone therapy and the results from follow-up examinations. ${ }^{12}$

\section{CONCLUSIONS}

Pleural effusion is a very uncommon manifestation of HNL that has rarely been reported in these patients. We presented a case of $\mathrm{HNL}$ with bilateral pleural effusion that was diagnosed using cervical lymph node biopsy and which was successfully treated with prednisone.

HNL patients are often admitted to a respiratory department, and it is easy to misdiagnose them as having tuberculous pleurisy or other diseases. We reported on this patient in order to improve clinicians' understanding of such diseases and help reduce the likelihood of misdiagnosis.

\section{REFERENCES}

1. Chen HC, Lai JH, Huang GS, et al. Systemic lupus erythematosus with simultaneous onset of Kikuchi-Fujimoto's disease complicated with antiphospholipid antibody syndrome: a case report and review of the literature. Rheumatol Int. 2005;25(4):303-6.

2. Papaioannou G, Speletas M, Kaloutsi V, Pavlitou-Tsiontsi A. Histiocytic necrotizing lymphadenitis (Kikuchi-Fujimoto disease) associated with antiphospholipid syndrome: case report and literature review. Ann Hematol. 2002;81(12):732-5.
3. Bosch X, Guilabert A, Miquel R, Campo E. Enigmatic KikuchiFujimoto disease: a comprehensive review. Am J Clin Pathol. 2004;122(1):141-52.

4. Santana A, Lessa B, Galrão L, Lima I, Santiago M. Kikuchi-Fujimoto's disease associated with systemic lupus erythematosus: case report and review of the literature. Clin Rheumatol. 2005;24(1):60-3.

5. Yu HL, Lee SS, Tsai HC, et al. Clinical manifestations of Kikuchi's disease in southern Taiwan. J Microbiol Immunol Infect. 2005;38(1):35-40.

6. Ito K, Morooka M, Kubota K. Kikuchi disease: 18F-FDG positron emission tomography/computed tomography of lymph node uptake. Jpn J Radiol. 2010;28(1):15-9.

7. Kuo TT. Kikuchi's disease (histocytic necrotizing lymphadenitis). A clinicopathologic study of 79 cases with an analysis of histologic subtypes, immunohistology, and DNA ploidy. Am J Surg Pathol. 1995;19(7):798-809.

8. Pileri SA, Facchetti F, Ascani S, et al. Myeloperoxidase expression by histiocytes in Kikuchi's and Kikuchi-like lymphadenopathy. Am J Pathol. 2001;159(3):915-24.

9. Ohshima K, Shimazaki K, Kume T, et al. Perforin and Fas pathways of cytotoxic T-cells in histiocytic necrotizing lymphadenitis. Histopathology. 1998;33(5):471-8.

10. Garcia-Zamalloa A, Taboada-Gomez J, Bernardo-Galán P, et al. Bilateral pleural effusion and interstitial lung disease as unusual manifestations of Kikuchi-Fujimoto disease: case report and literature review. BMC Pulm Med. 2010;10:54.

11. Jun-Fen F, Chun-Lin W, Li L, et al. Kikuchi-Fujimoto disease manifesting as recurrent thrombocytopenia and Mobitz type II atrioventricular block in a 7-year-old girl: a case report and analysis of 138 Chinese childhood Kikuchi-Fujimoto cases with 10 years of follow-up in 97 patients. Acta Paediatr. 2007;96(12):1844-7.

12. Jang YJ, Park KH, Seok HJ. Management of Kikuchi's disease using glucocorticoid. J Laryngol Otol. 2000;114(9):709-11.

Sources of funding: None

Conflict of interest: The authors declare that they have no conflict of interest

Date of first submission: December 10, 2016

Last received: January 23, 2017

Accepted: February 17, 2017

Address for correspondence:

Xuchun Liu

Department of Respiratory Medicine, Chizhou People's Hospital,

Chizhou, Anhui 247100, China

E-mail: liuxu0832@sina.com 